EPJ Web of Conferences 78, 01001 (2014)

DOI: $10.1051 /$ epjconf/20147801001

(C) Owned by the authors, published by EDP Sciences, 2014

\title{
Relativistic Wigner functions
}

\author{
Iwo Bialynicki-Birula ${ }^{a}$ \\ Center for Theoretical Physics, Lotników 32/46, Warsaw, Poland
}

\begin{abstract}
Original definition of the Wigner function can be extended in a natural manner to relativistic domain in the framework of quantum field theory. Three such generalizations are described. They cover the cases of the Dirac particles, the photon, and the full electromagnetic field.
\end{abstract}

\section{Introduction}

The difficulties in extending the concept of the Wigner function to the relativistic domain were often considered unsurmountable. In a review paper by M. Hillery, R. F. O'Connell, M. O. Scully, and E. P. Wigner we find a rather pessimistic opinion on this subject [1]: "It (the Wigner function) is non-relativistic in nature because it is not invariant under the Lorentz group; also configuration space quantum mechanics for more than one particle would be difficult to formulate relativistically".

Of course, owing to the processes of particle creation and annihilation relativistic quantum mechanics does not exist as a fully consistent theory and it must be replaced by quantum field theory. Nevertheless the original construction of Wigner (and Szilard) can be extended, as has been shown long time ago [2], in a natural manner to quantum field theory just by replacing wave functions by the expectation values of field operators. The expectation value in a one-particle state of such an object reduces in the nonrelativistic approximation to the standard Wigner function. From this observation there is a natural generalization to a relativistic quantum field theory. Of course, in full quantum field theory the evolution equations for the Wigner function involves higher order functions that are built from products of more than two field operators and the hierarchy of such equations does not close. However, when the coupling to the external field plays the dominant role and the mutual particle interactions can be neglected, one obtains the evolution equation similar to the transport equation for the standard Wigner function. The most interesting case of such a field-theoretic generalization of the Wigner function, called the Dirac-Heisenberg-Wigner (DHW) function, is obtained for the electronpositron field [3]. One important application of this construction is the description of electron-positron pair creation by an applied electromagnetic field. The advantage of using the DHW function instead more standard methods is that one can avoid the complications arising from the many-body character of relativistic quantum field theory. It is sufficient to start from a "good" Wigner function to guarantee that its time evolution will always take us to a physical function. The corresponding evolution equations in some cases can be even solved analytically.

Every quantum field theory allows for the construction of the Wigner function from field operators but for the electromagnetic field (and also for other bosonic fields) there is also an entirely different

ae-mail: birula@cft.edu.pl 
construction that leads to a Wigner-type functional that is a natural generalization of the standard Wigner function. Such a functional was derived for the scalar field in [4]. However, the electromagnetic case seems to be more interesting. The arguments of the electromagnetic Wigner functional are the electric and magnetic fields and not the points in the phase space, as in the quantum-mechanical case. Still, this functional shares many properties with the Wigner function and it enables one to learn something new about the properties of the quantized electromagnetic field. In particular, this construction gives a new insight into the statistical properties of the electromagnetic field at finite temperature.

\section{Dirac-Heisenberg-Wigner function}

The starting point for the construction of the Wigner function is the expectation value of the product of field operators of the Dirac field $\left\langle\Phi\left|\hat{\psi}_{\alpha}(\vec{r}, t), \hat{\psi}_{\beta}^{\dagger}\left(\vec{r}^{\prime}, t\right)\right| \Phi\right\rangle$. This object has $4 \times 4=16$ components. Such expectation values were studied by Dirac [5] and Heisenberg [6] but without the connection to the Wigner function. I shall only consider the Dirac field interacting with the external electromagnetic field $\vec{A}(\vec{r}, t)$. The final definition of the Wigner function involves two adjustments. First, the product of two field operators can be written as the sum of the commutator and the anticommutator divided by 2 . However, the anticommutator can be disregarded because it does not carry any useful information. According to canonical commutation relations it is just a delta function and one is left with the commutator. Second, the requirement of the gauge invariance of the Wigner function is fulfilled by the insertion of the line integral in the exponent that compensates for the changes of the phases of the Dirac field operators. Thus, the final expression for the 16-component Wigner function introduced in [3] is:

$$
W_{\alpha \beta}(\vec{r}, \vec{p}, t)=-\frac{1}{2} \int d^{3} \eta e^{2 i \vec{p} \cdot \vec{\eta} / \hbar} e^{-i e \int_{-1}^{1} d \lambda \vec{\eta} \cdot \vec{A}(\vec{r}+\lambda \vec{\eta}, t)}\left\langle\Phi\left|\left[\hat{\psi}_{\alpha}(\vec{r}-\vec{\eta}, t), \hat{\psi}_{\beta}^{\dagger}(\vec{r}+\vec{\eta}, t)\right]\right| \Phi\right\rangle .
$$

Even in the simple case when only the external electromagnetic field is taken into account the evolution equations resulting from the Dirac equation obeyed by the Dirac field operators are quite complex. They are written below in the approximation that the electromagnetic field is macroscopic, i.e. it does not change appreciably on the microscopic space and time scale $\left(3.8 \times 10^{-13} \mathrm{~m}\right.$ and $\left.1.3 \times 10^{-21} \mathrm{~s}\right)$ characteristic of electrons. The 16 components of the Wigner functions are grouped into four scalars functions $f_{0}, f_{1}, f_{2}, f_{3}$ and four three-dimensional vector functions $\vec{g}_{0}, \vec{g}_{1}, \vec{g}_{2}, \vec{g}_{3}$ of the phase-space variables $\vec{r}, \vec{p}$ and $t$.

$$
\begin{aligned}
\mathrm{D}_{t} f_{0}+c \overrightarrow{\mathrm{D}} \cdot \vec{g}_{1} & =0 \\
\mathrm{D}_{t} f_{1}+c \overrightarrow{\mathrm{D}} \cdot \vec{g}_{0} & =-2 \frac{m c^{2}}{\hbar} f_{2}, \\
\mathrm{D}_{t} f_{2}+2 \frac{c}{\hbar} \vec{p} \cdot \vec{g}_{3} & =2 \frac{m c^{2}}{\hbar} f_{1}, \\
\mathrm{D}_{t} f_{3}-2 \frac{c}{\hbar} \vec{p} \cdot \vec{g}_{2} & =0 \\
\mathrm{D}_{t} \vec{g}_{0}+c \overrightarrow{\mathrm{D}} f_{1}-2 \frac{c}{\hbar} \vec{p} \times \vec{g}_{1} & =0 \\
\mathrm{D}_{t} \vec{g}_{1}+c \overrightarrow{\mathrm{D}} f_{0}-2 \frac{c}{\hbar} \vec{p} \times \vec{g}_{0} & =-2 \frac{m c^{2}}{\hbar} \vec{g}_{2} \\
\mathrm{D}_{t} \vec{g}_{2}+c \overrightarrow{\mathrm{D}} \times \vec{g}_{3}+2 \frac{c}{\hbar} \vec{p} f_{3} & =2 \frac{m c^{2}}{\hbar} \vec{g}_{1}, \\
\mathrm{D}_{t} \vec{g}_{3}-c \overrightarrow{\mathrm{D}} \times \vec{g}_{2}-2 \frac{c}{\hbar} \vec{p} f_{2} & =0
\end{aligned}
$$


where

$$
\begin{aligned}
\mathrm{D}_{t} & =\partial_{t}+e \vec{E}(\vec{r}, t) \cdot \overrightarrow{\partial_{p}}, \\
\overrightarrow{\mathrm{D}} & =\vec{\nabla}+e \vec{B}(\vec{r}, t) \times \overrightarrow{\partial_{p}} .
\end{aligned}
$$

The components of the DHW function have the following physical interpretation:

$f_{0}$ - charge density

$f_{3}$ - mass density

$\vec{g}_{0}$ - spin density

$\vec{g}_{1}$ - current density

$\vec{g}_{3}$ - magnetic moment density

The remaining components do not have a simple interpretation.

The evolution equations $(2 \mathrm{a}-2 \mathrm{~h})$ are universal. They determine the time evolution of the Wigner function for every choice of the state $|\Psi\rangle$ and also for mixed states. However, one must make sure that the initial values represent an allowed choice because not every set of functions corresponds to a genuine Wigner function. This is also true in nonrelativistic quantum mechanics for the standard Wigner function. In our case there is one simple choice when the expectation value can be evaluated: the vacuum state in the absence of the electromagnetic field. The only nonvanishing components for the vacuum state are:

$$
f_{3}=-\frac{2 m c}{\sqrt{\vec{p}^{2}+m^{2} c^{2}}}, \quad \vec{g}_{1}=-\frac{2 \vec{p}}{\sqrt{\vec{p}^{2}+m^{2} c^{2}}} .
$$

With the choice of the vacuum as the initial state the evolution equations can be solved exactly in some simple cases, for example, when the electric field is only a function of time [7,8]. With the help of these solutions it was possible to prove, in particular, that the famous Schwinger essential singularity in the formula for the number of pairs produces by the constant electric field disappears for the electric field $[8,9]$ that has been switched on in the remote past.

The analytic solution of the evolution equations (2) was possible for a uniform electric field because these equations reduce in this case to a set of three equations that have, in addition, a form of equations for a precessing top. By choosing appropriate combinations of the components of the DHW matrix, one obtains the following three coupled evolution equations:

$$
\left(\partial_{\tau}+\mathcal{E}(\tau) \partial_{q}\right)\left[\begin{array}{l}
w_{1} \\
w_{2} \\
w_{3}
\end{array}\right]=2\left[\begin{array}{ccc}
0 & q & 0 \\
-q & 0 & 1 \\
0 & -1 & 0
\end{array}\right]\left[\begin{array}{l}
w_{1} \\
w_{2} \\
w_{3}
\end{array}\right],
$$

These equations by the substitution $w_{i}(q, \tau)=\exp \left(\mathcal{A}(\tau) \partial_{q}\right) \mathcal{X}_{i}(q, \tau)=\mathcal{X}_{i}(q+\mathcal{A}(\tau), \tau)$ are transformed into a set of ordinary differential equations,

$$
\frac{d}{d \tau}\left[\begin{array}{l}
X_{1} \\
\mathcal{X}_{2} \\
\mathcal{X}_{3}
\end{array}\right]=2\left[\begin{array}{ccc}
0 & q-\mathcal{A}(\tau) & 0 \\
-q+\mathcal{A}(\tau) & 0 & 1 \\
0 & -1 & 0
\end{array}\right]\left[\begin{array}{l}
X_{1} \\
\mathcal{X}_{2} \\
\mathcal{X}_{3}
\end{array}\right]
$$

where $\mathcal{A}(\tau)$ is the (dimensionless) potential of the electric field $-\partial_{\tau} \mathcal{A}(\tau)=\mathcal{E}(\tau)$. After rewriting these equations in the vectorial notation one recognizes the evolution equation of a spinning top

$$
\frac{d \mathcal{X}(q, \tau)}{d \tau}=-2 \mathcal{H}(q, \tau) \times \mathcal{X}(q, \tau)
$$

where $\mathcal{H}(\tau)=(1,0, q-\mathcal{A}(\tau))$. The reduction of these three equations to a single equation produces a rather complicated third-order equation. Third-order equations are much harder to solve as compared 
to second-order equations. Fortunately, the group of rotations that underlines the equation (7) enables one to replace these equations by an equivalent set of two first-order equations that lead to a secondorder equation. To realize this program, one may observe that every real vector $\mathcal{X}$ can be expressed in terms of a two-component spinor $\Psi=\left(\psi_{-}, \psi_{+}\right)$with the use of Pauli matrices, $\mathcal{X}=\Psi^{\dagger} \boldsymbol{\sigma} \Psi$. One may then check that if $\Psi$ satisfies the equation:

$$
\frac{d \Psi(q, \tau)}{d \tau}=i \sigma \cdot \mathcal{H}(q-\mathcal{A}(\tau)) \Psi(q, \tau)
$$

then $\mathcal{X}=\Psi^{\dagger} \boldsymbol{\sigma} \Psi$ satisfied the equation (7). The pair of first-order equations (8) leads to two separate second-order equations,

$$
\left[\frac{d^{2}}{d \tau^{2}}+1+(q-\mathcal{A}(\tau))^{2} \mp i \mathcal{E}(\tau)\right] \psi_{\mp}(q, \tau)=0 .
$$

These equations possess exact analytic solutions for several special choices of the electric field. In particular, such solutions exist in the following cases:

$$
\mathcal{E}(\tau)=\mathcal{E}_{0} e^{b \tau}, \quad \mathcal{E}(\tau)=\mathcal{E}_{0} / \tau^{2}, \quad \mathcal{E}(\tau)=\mathcal{E}_{0} / \cosh b \tau
$$

In each case the electric field vanishes in the remote past. Therefore, one may assume that the state at $t=-\infty$ was the free vacuum state whose Wigner function is given by (4). Analytic properties of the solutions exclude a singular behavior at $\mathcal{E}_{0}=0$. This result can be extended [9] to a wide class of electric fields. The only condition is that the perturbative expansions for these fields are majorized by some analytic solution. Thus, the famous Schwinger essential singularity [14] is due to an infinite duration of the field. Time-independent electric field has infinitely long time to give virtual particles arbitrarily high momenta,leading to a singular behaviour.

\section{Wigner function for the photon}

The construction of the Wigner function for the photon can proceed along the same lines as for the electrons. The adequate counterpart of the Dirac field operator $\hat{\psi}$ is the Riemann-Silberstein vector $\vec{F}$ $[10,11]$

$$
\vec{F}(\vec{r}, t)=\frac{\vec{D}(\vec{r}, t)}{\sqrt{2 \epsilon_{0}}}+i \frac{\vec{B}(\vec{r}, t)}{\sqrt{2 \mu_{0}}},
$$

The analogy with the Dirac field is based on the same general form of the evolution equation,

$$
i \partial_{t}(\vec{r}, t)=\nabla \times \vec{F}(\vec{r}, t) .
$$

The vector $\vec{F}$ also must satisfy the subsidiary condition $\nabla \cdot \vec{F}=0$.

The photon Wigner function is a $3 \times 3$ matrix defined as:

$$
W_{i j}(\vec{r}, \vec{k}, t)=\frac{1}{2} \int d^{3} \eta e^{2 i \vec{k} \cdot \vec{\eta}}\left\langle\Phi\left|\left\{\hat{F}_{i}(\vec{r}-\vec{\eta}, t), \hat{F}_{j}^{\dagger}(\vec{r}+\vec{\eta}, t)\right\}\right| \Phi\right\rangle,
$$

where $\hat{F}_{i}(\vec{r})$ and $\hat{F}_{j}^{\dagger}(\vec{r})$ are the field operators corresponding to the components of the RiemannSilberstein vector and its conjugate. The product of field operators was replaced by an anticommutator since the commutator is just a number. The hermitian matrix $W_{i j}$ can be parametrized by 9 real functions: a scalar $w$, a traceless symmetric matrix $w_{i j}$ and a vector $u_{i}$,

$$
W_{i j}(\vec{k}, \vec{r}, t)=\frac{1}{3} w(\vec{k}, \vec{r}, t) \delta_{i j}+w_{i j}(\vec{k}, \vec{r})+\frac{c}{2 i} \varepsilon_{i j k} u_{k}(\vec{k}, \vec{r}, t) .
$$


On account of of the subsidiary condition satisfied by $\vec{F}$, the evolution equations for $w$ and $u_{i}$ decouple from $w_{i j}$ and take on a simple form [10],

$$
\partial_{t} w(\vec{k}, \vec{r}, t)=-c^{2} \vec{\nabla} \cdot \vec{u}(\vec{k}, \vec{r}, t), \quad \partial_{t} \vec{u}(\vec{k}, \vec{r}, t)=-2 c \vec{k} \times \vec{u}(\vec{k}, \vec{r}, t)-\vec{\nabla} w(\vec{k}, \vec{r}, t) .
$$

These equations may be viewed as the transport equation of the energy density $w$ and the momentum density $\vec{u}$ in the phase space. An interesting feature of these equations is the precession of the momentum density around the vector $\vec{k}$. As was the case in the DHW function for the electron field, the equations (15) are universal; they have the same form for every state vector $|\Phi\rangle$.

\section{Wigner functional for the electromagnetic field}

The electromagnetic field can be viewed as a huge (infinite-dimensional) harmonic oscillator. Therefore, one can construct the Wigner function for the whole field by the straightforward analogy with the harmonic oscillator [12]. To this end one may observe that the Wigner function for the ground state of the one-dimensional harmonic oscillator $W_{G}(p, x)$ is directly related to the Hamiltonian,

$$
W_{G}(p, x)=\frac{1}{\pi \hbar} \exp \left(-\frac{2 H(p, x)}{\hbar \omega}\right), \quad H(p, x)=\frac{p^{2}}{2 m}+\frac{m \omega^{2} x^{2}}{2} .
$$

Since $\hbar \omega$ is the energy of a single excitation of the oscillator, the exponent is simply twice the number of excitations. This observation makes it possible to write down the expression for the Wigner functional of the electromagnetic field. All one needs is the formula for the number of excitations, i.e. the number of photons $N[\vec{D}, \vec{B}]$. Such a formula has been given long time ago by Zeldovich [13] and it reads:

$$
N[\vec{D}, \vec{B}]=\frac{1}{4 \pi^{2} \hbar c} \int d^{3} r \int d^{3} r^{\prime}\left[\frac{\vec{D}(\vec{r}) \cdot \vec{D}\left(\vec{r}^{\prime}\right) / \epsilon+\vec{B}(\vec{r}) \cdot \vec{B}\left(\vec{r}^{\prime}\right) / \mu}{\left|\vec{r}-\vec{r}^{\prime}\right|^{2}}\right] .
$$

It can be obtained from the energy (Hamiltonian) of the electromagnetic field $H[\vec{D}, \vec{B}]$,

$$
H[\vec{D}, \vec{B}]=\frac{1}{2} \int d^{3} r\left[\frac{\vec{D}(\vec{r}) \cdot \vec{D}(\vec{r})}{\epsilon}+\frac{\vec{B}(\vec{r}) \cdot \vec{B}(\vec{r})}{\mu}\right] .
$$

To obtain $N[\vec{D}, \vec{B}]$ from $H[\vec{D}, \vec{B}]$ one may observe that the counterpart of $\hbar \omega$ in the formula (16) for the one dimensional oscillator is the Fourier transform that takes care of all the modes of the electromagnetic field,

$$
\frac{1}{\hbar \omega} \rightarrow \int \frac{d^{3} k}{(2 \pi)^{3}} \frac{e^{i \vec{k} \cdot\left(\vec{r}-\vec{r}^{\prime}\right)}}{\hbar c k}=\frac{1}{2 \pi^{2} \hbar c\left|\vec{r}-\vec{r}^{\prime}\right|^{2}}
$$

For the ground state (the vacuum) of the electromagnetic field one obtains the following Wigner functional:

$$
W[\vec{D}, \vec{B}]=\exp (-2 N[\vec{D}, \vec{B}])=\exp \left\{-\frac{1}{2 \pi^{2} \hbar c} \int d^{3} r \int d^{3} r^{\prime}\left[\frac{\vec{D}(\vec{r}) \cdot \vec{D}\left(\vec{r}^{\prime}\right) / \epsilon+\vec{B}(\vec{r}) \cdot \vec{B}\left(\vec{r}^{\prime}\right) / \mu}{\left|\vec{r}-\vec{r}^{\prime}\right|^{2}}\right]\right\}
$$

There is one property of this functional that is lost in the transition from harmonic oscillators to the full electromagnetic field: the normalization. The prefactor in the Wigner function for the $n$-dimensional harmonic oscillator is $(1 / \pi \hbar)^{n}$ and it clearly blows up for an infinite number of dimensions $(n \rightarrow \infty)$. Therefore, the formula (20) can only be used to calculate relative probabilities. Still, the Wigner 
functional of the vacuum contains the information that is not easily seen in the standard formulation. For example, one may conclude immediately from the formulas (17) and (20) that the field distribution is Gaussian. To establish this fact in the standard approach one would have to calculate all field correlation functions. Moreover, one can see that the ground state (the vacuum) of the electromagnetic field exhibits "antiferromagnetic" properties. Namely, it is more probable to find in the vicinity of a point $\vec{r}$ the fields that are pointing in the opposite direction $\left(\vec{D}(\vec{r}) \cdot \vec{D}\left(\vec{r}^{\prime}\right)<0\right)$ rather than those pointing in the same direction $\left(\vec{D}(\vec{r}) \cdot \vec{D}\left(\vec{r}^{\prime}\right)>0\right)$

Finally, I would like to show how the analogy with the simple harmonic oscillator enables one to find without a substantial effort the statistical properties of the electromagnetic field at finite temperature. To this end, I shall start with the Wigner function $W_{T}(p, x)$ for the one-dimensional harmonic oscillator at finite temperature. The density operator for the thermal state is $\rho=e^{-\frac{A}{k_{B} T}} / \operatorname{Tr}\left\{e^{-\frac{A}{k_{B} T}}\right\}$. The calculation of the Wigner function involves the summation over all energy states [1] and it produces the formula:

$$
W_{T}(x, p)=\frac{1}{\pi \hbar} \tanh \left(\frac{\hbar \omega}{2 k_{B} T}\right) \exp \left[-2 \tanh \left(\frac{\hbar \omega}{2 k_{B} T}\right) \frac{H(p, x)}{\hbar \omega}\right] .
$$

I shall now proceed, as in the case of the ground state, and I replace the function of $\omega$ by the appropriate Fourier transform,

$$
\frac{1}{\hbar \omega} \tanh \left(\frac{\hbar \omega}{2 k_{B} T}\right) \rightarrow \int \frac{d^{3} k}{(2 \pi)^{3}} \frac{e^{i \vec{k} \cdot\left(\vec{r}-\vec{r}^{\prime}\right)}}{\hbar c k} \tanh \left(\frac{l_{T} k}{2}\right)=\frac{1}{\pi \hbar^{2} c^{2} l_{T}\left|\vec{r}-\vec{r}^{\prime}\right| \sinh \left(\left|\vec{r}-\vec{r}^{\prime}\right| / l_{T}\right)},
$$

where $l_{T}=h c / k_{B} T$ (it is $h$ and not $\hbar$ ) is the thermal length. Of course, in the limit, when $T \rightarrow 0$, one recovers the previous result (19). At a finite temperature one finds an exponential decrease of correlations at the distance of the order of thermal length. The final formula for the Wigner functional of the electromagnetic field at the temperature $T$ is:

$$
W_{T}[\vec{D}, \vec{B}]=\exp \left[-\frac{2}{\hbar c} \int d^{3} r \int d^{3} r^{\prime} \frac{\vec{D}(\vec{r}) \cdot \vec{D}\left(\vec{r}^{\prime}\right) / \epsilon+\vec{B}(\vec{r}) \cdot \vec{B}\left(\vec{r}^{\prime}\right) / \mu}{l_{T}\left|\vec{r}-\vec{r}^{\prime}\right| \sinh \left(\left|\vec{r}-\vec{r}^{\prime}\right| / l_{T}\right)}\right] .
$$

Another example where the Wigner functional is easily found is a coherent state. In the case of the harmonic oscillator this Wigner function is the displaced ground state function,

$$
W_{c}(p, x)=\frac{1}{\pi \hbar} \exp \left(-\frac{\left(p-\mathcal{P}_{c}\right)^{2} / 2 m+m \omega^{2}\left(x-\mathcal{X}_{c}\right)^{2} / 2}{\hbar \omega}\right)
$$

where $\mathcal{X}_{c}$ and $\mathcal{P}_{c}$ are directly related to the real and imaginary parts of the coherent state amplitude $\alpha$,

$$
\alpha=\mathcal{X}_{c} \sqrt{\frac{m \omega}{2 \hbar}}+i \mathcal{P}_{c} \sqrt{\frac{1}{2 \hbar m \omega}} .
$$

Of course, $\mathcal{X}_{c}$ and $\mathcal{P}_{c}$ are nothing else but the expectation values of the position and momentum operators in the coherent state. Using the result for the harmonic oscillator one can immediately write down the Wigner functional for the coherent state of the electromagnetic field by displacing the arguments in the formula (17) for the number of photons

$$
W_{c}[\vec{D}, \vec{B}]=\exp \left\{-\frac{1}{2 \pi^{2} \hbar c} \int d^{3} r \int d^{3} r^{\prime}\left[\frac{\Delta \vec{D}(\vec{r}) \cdot \Delta \vec{D}\left(\vec{r}^{\prime}\right) / \epsilon+\Delta \vec{B}(\vec{r}) \cdot \Delta \vec{B}\left(\vec{r}^{\prime}\right) / \mu}{\left|\vec{r}-\vec{r}^{\prime}\right|^{2}}\right]\right\},
$$

where $\Delta \vec{D}(\vec{r})=\vec{D}(\vec{r})-\overrightarrow{\mathcal{D}}_{c}(\vec{r})$ and $\Delta \vec{B}(\vec{r})=\vec{B}(\vec{r})-\overrightarrow{\mathcal{B}}_{c}(\vec{r})$ are the fields displaced by the expectation values $\overrightarrow{\mathcal{D}}_{c}(\vec{r})$ and $\overrightarrow{\mathcal{B}}_{c}(\vec{r})$ in the coherent state. The Gaussian Wigner function is positive and can 
be treated as a bona fide probability distribution. The formula (26) shows that the probability to find various field values attains the maximum value at the classical coherent-state values and then decreases exponentially when we move away from the maximum.

\section{Summary}

I have illustrated the concept of the relativistic Wigner function with three different examples. I hope that these examples conveyed the main message of this work: the extension of the Wigner function to relativistic domain gives a new look at some problems and also might simplify the analysis. At first, it might seem that the definition of the Wigner involving integrals evaluated at equal-time blatantly violates the relativistic notion of the unity of space and time. There were many efforts [15-17]to correct this seeming shortcoming by including also integration over time. However, such constructions turned out not to be very useful because they requires the knowledge of the whole history of the system. This information is availably only for a completely solvable system but in this case there is little advantage of using the Wigner function. In contrast, the Wigner functions given by the formulas (1) and (13) determine the time evolution once their initial values are specified. It is true that these Wigner functions do not have a relativistic form; their transformation properties under the Lorentz transformations are horrible. Still, the results obtained with their use do not violate the laws of relativistic physics since at the foundation of the whole procedure lies a fully relativistic theory (Dirac equation, Maxwell equations, etc.). It is only the explicit Lorentz covariance that is missing.

\section{Acknowledgments}

This research was partly supported by the grant from the Polish Ministry of Science and Higher Education for the years 2013-2016.

\section{References}

[1] M. Hillery, R. F. O’Connell, M. O. Scully, and E. P. Wigner, Physics Reports 106, 121 (1984).

[2] L. P. Kadanoff and G. Baym, Quantum Statistical Mechanics (Benjamin, New York, 1962), p. 67.

[3] I. Bialynicki-Birula, P. Górnicki, and J. Rafelski, Phys. Rev. D 44, 1825 (1991).

[4] S. Mrówczynski and B. Miller, Phys. Rev. D 50, 7542 (1996).

[5] P. A. M. Dirac, Proc. Cambridge Philos. Soc. 30, 150 (1934).

[6] W. Heisenberg, Z. Phys. 90, 209 (1934).

[7] I. Bialynicki-Birula and Ł. Rudnicki, Phys. Rev. D 83, 065020 (2011).

[8] I. Bialynicki-Birula, Ł. Rudnicki and A. Wienczek 2011 arXiv:1108.2615.

[9] I. Bialynicki-Birula and Ł. Rudnicki, to be published.

[10] I. Bialynicki-Birula, Photon wave function Progress in Optics, edited by E. Wolf (Elsevier, Amsterdam, 1996), Vol. 36; see also ArXiv: quant-ph/0508202).

[11] I. Bialynicki-Birula and Z. Bialynicka-Birula, J. Phys. A 46, 053001 (2013).

[12] I. Bialynicki-Birula, Opt. Comm. 179, 237 (2000).

[13] Ya. B. Zeldovich, Dokl. Acad. Sci. USSR 163, 1359 (1965), in Russian.

[14] J. Schwinger J, Phys.Rev. 82, 664 (1951).

[15] P. Zhuang and U. Heinz, Phys. Rev. 53, 2096 (1996).

[16] A. Abada, M. C. Birse, P. Zhuang, and U. Heinz, Phys. Rev. 54, 4175 (1996).

[17] V. G. Morozov, G. Röpke, and A. Höll, Theor. Math. Phys. 131, 812 (2002). 
